\title{
The influence of electromagnetic singularities on an active dipole antenna within a cavity
}

\author{
F. Gronwald \\ Institute for Fundamental Electrical Engineering and EMC, Otto-von-Guericke University Magdeburg, P.O. Box 4120, 39016 \\ Magdeburg, Germany
}

\begin{abstract}
We study the influence of both the electromagnetic source singularity and electromagnetic cavity resonances on the current distribution of a dipole antenna within a rectangular cavity. These two types of electromagnetic singularities are triggered by the radius of the dipole antenna and the quality factor of the enclosing cavity, respectively. The key element of our investigation is a novel representation of the electromagnetic Green's function for a lossy rectangular cavity. It allows to directly obtain the current distribution on the antenna by means of the method of moments. As a result it is recognized that a dominating source singularity, i.e., a small antenna radius, can inhibit resonating effects, even if a cavity resonance is excited and the quality factor of the cavity is high.
\end{abstract}

\section{Introduction}

Many EMC problems involve the investigation of electromagnetic coupling within metallic or resonating structures (Tesche et al., 1997; Lee, 1995). This is obvious for electric and electronic devices that are inside of a metallic enclosure and appear as victims of Electromagnetic Interference (EMI). Also complex systems, like aircrafts or cars, for example, often contain sensitive electric and electronic equipment within resonating metallic compartments. In these cases the electromagnetic interference process can considerably differ from that in free space. This difference can be explained by the notion of electromagnetic singularities: If we consider an electric charge distribution we encounter the well-known singularity in the source region which is due to the singular behavior of the Coulomb field. This type of singularity is present both if the charge distribution is located in free space and if the charge distribution is enclosed by a cavity. Therefore the

Correspondence to: F. Gronwald

(Frank.Gronwald@et.uni-magdeburg.de) mathematical methods for dealing with the singularity in the source region are the same for both free space and the inside of a cavity. A difference between free space and the inside of a cavity occurs, however, if radiation fields are considered. In free space, radiation fields carry a finite amount of energy and exhibit no singularities. If radiation fields are enclosed by a cavity they form discrete bound states, the modes. In frequency domain the energy of a mode is bounded by the quality factor of the cavity and tends to infinity in the lossless case. This singular behavior of a resonance has no analogue in free space.

Since the electromagnetic coupling gets very strong close to an electromagnetic singularity it is clear that the strength of a singularity will largely determine the behavior of an EMC-relevant coupling process within a cavity. In this context it needs to be mentioned that besides the quality factor, which triggers the strength of a resonance, there will be a second cut-off parameter which triggers the source singularity. In our model, where we focus on a dipole antenna, this parameter will be given by the antenna radius. It is then interesting to observe the effect of varying the quality factor or the antenna radius. It will be recognized that a strong source singularity, corresponding to a small wire radius, will qualitatively impress a "free space behavior" of electromagnetic quantities while a strong resonance, corresponding to a high quality factor, will lead to a resonating behavior which does not resemble that of free space.

In the next Sect. 2 we will introduce the model of an active dipole antenna within a rectangular cavity and outline how to establish an appropriate integral equation for the antenna current. The influence of the quality factor and the antenna radius on the Green's function of the cavity will be studied. Finally, in Sect. 3, the antenna current on the dipole antenna will be obtained by the method of moments and subsequently be discussed. 


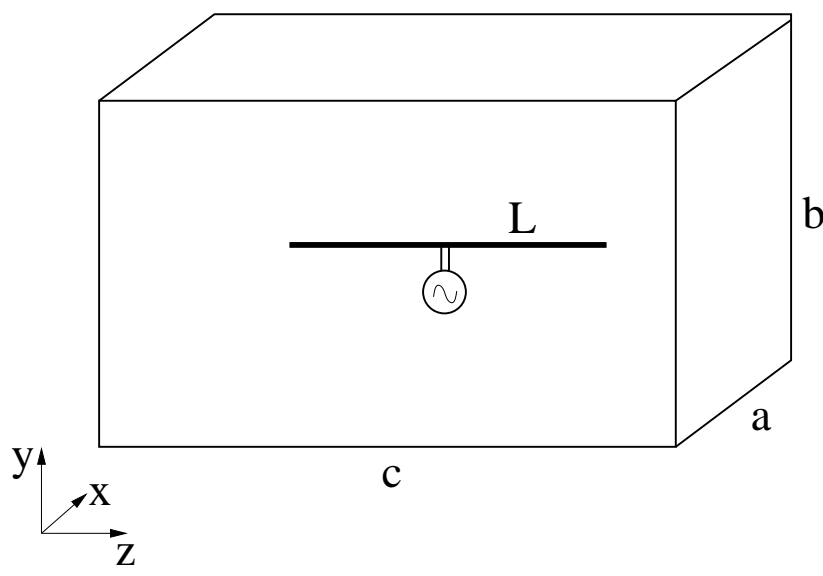

Fig. 1. Sketch of the dipole antenna within the rectangular cavity.

\section{Hallén's integral equation and the Green's function of the cavity}

There are two standard integral equations which can be used to determine the current on a wire antenna. These are Pocklington's equation and Hallén's equation (Nakano, 1996). In the following we focus on Hallén's equation since it contains the Green's function $G^{A}$ for the vector potential as kernel. This kernel is easier to calculate than the one of Pocklington's equation. It is one of our goals to efficiently solve Hallén's equation within a rectangular cavity by means of the method of moments.

We consider a center fed, straight dipole antenna, compare Fig. 1. In a cartesian coordinate system we align the antenna with the $z$-axis and position the center of the antenna at $z=$ $z_{0}$. The antenna is excited by a slice generator that is characterized by the electric source field $E_{z}^{q}(z)=V_{0} \delta\left(z-z_{0}\right)$. If furthermore a thin-wire approximation is employed it is found that Hallén's equation acquires the form

$$
\begin{aligned}
& \int_{-L / 2}^{L / 2} G^{A}\left(z, z^{\prime}\right) I\left(z^{\prime}\right) d z^{\prime}= \\
& \quad-\frac{j}{\eta}\left(A \cos \left(k\left(z-z_{0}\right)\right)+\frac{V_{0}}{2} \sin \left(k\left|z-z_{0}\right|\right)\right) .
\end{aligned}
$$

Here the symbol $\eta$ denotes the intrinsic impedance of the surrounding medium, $\eta=\sqrt{\mu / \varepsilon}$, and $k$ denotes the possibly complex wavenumber, $k=\omega / c$. The integral extends over the length of the antenna which is denoted by $L$. In Eq. (1) the unknowns are the current distribution $I(z)$ and the integration constant $A$. These unknowns are to be determined by the method of moments.

If the antenna is placed in free space the Green's function $G^{A}\left(z, z^{\prime}\right)$ is given by the Green's function of free space. Due to the thin-wire approximation, which is valid if the wire radius $\rho$ is much smaller than the wavelength $\lambda, \rho \ll \lambda$, we have $G^{A}\left(z, z^{\prime}\right) \longrightarrow G_{\text {red }}^{A}\left(z, z^{\prime}\right)$ with the "reduced kernel"

$G_{\mathrm{red}}^{A}\left(z, z^{\prime}\right)=\frac{1}{4 \pi} \frac{e^{-j k \sqrt{\rho^{2}+\left(z-z^{\prime}\right)^{2}}}}{\sqrt{\rho^{2}+\left(z-z^{\prime}\right)^{2}}}$.
If the antenna is not placed in free space but put within a cavity it still is possible to obtain the current distribution $I(z)$ from Hallén's equation Eq. (1). However, then it is necessary to use the Green's function of the cavity as kernel, i.e., $G^{A}\left(z, z^{\prime}\right) \longrightarrow G_{\mathrm{cav}}^{A}\left(z, z^{\prime}\right)$ (Tai, 1994).

For a given cavity it is of paramount importance to find a representation of the Green's function $G_{\text {cav }}^{A}\left(z, z^{\prime}\right)$ which is numerically accurate and efficient in both the source region, where $z \longrightarrow z^{\prime}$, and the resonance region. The standard mode-representation usually fails to be efficient in the source region where convergence becomes very slow. Complementary to the mode-representation is the so-called rayrepresentation. It has good convergence properties in the source region but usually fails to be efficient in the resonance region. The complementary behavior of mode- and rayrepresentation already has been discussed in detail (Felsen, 1984). There the usage of hybrid ray-mode-representations was promoted in order to combine the mutual advantages of both ray- and mode-representation. For the case of a rectangular cavity an explicit ray-mode-representation was investigated (Wu and Chang, 1988) and already exhibited several advantages. In a different approach a ray-moderepresentation for a rectangular cavity was constructed (Park et al., 1988) on the basis of the Ewald sum technique (Ewald, 1921). A comparison has shown that the latter representation is extremely accurate and efficient if compared to other representations (Gronwald et al., 2002). However, the advantageous representation of Park et al. (1988) is not valid for complex wavenumbers. Therefore we will base in the following our numerical analysis on a representation of the Green's function of a rectangular cavity which generalizes this representation to complex wavenumbers (Gronwald, 2002).

For the electromagnetic vector potential the dyadic Green's function of a rectangular cavity, which is aligned with the $x-, y-$ and $z$-axis and is of dimensions $a, b$, and $c$, respectively, is of the form

$\overline{\bar{G}}^{A}=G_{x x}^{A} \hat{x} \hat{x}+G_{y y}^{A} \hat{y} \hat{y}+G_{z z}^{A} \hat{z} \hat{z}$.

Let us focus for concreteness on the $G_{z z}^{A}$ component. It was shown (Gronwald, 2002) that this component can be written in the form

$G_{z z}^{A}=G_{z z 1}^{A}+G_{z z 2}^{A}$

where

$$
\begin{gathered}
G_{z z 1}^{A}=\frac{\mu}{8 a b c} \sum_{m, n, p=-\infty}^{\infty} \sum_{i=0}^{7} A_{i}^{z z} \frac{\exp \left(-\frac{k_{0}^{2}-k^{2}}{4 E^{2}}\right)}{k_{0}^{2}-k^{2}} \\
\exp \left(j\left(k_{0 x} X_{i}+k_{0 y} Y_{i}+k_{0 z} Z_{i}\right)\right)
\end{gathered}
$$

and

$$
\begin{aligned}
& G_{z z 2}^{A}=\frac{\mu}{8 \pi} \sum_{m, n, p=-\infty}^{\infty} \sum_{i=0}^{7} A_{i}^{z z} \\
& {\left[\frac{\exp \left(j k R_{i, m n p}\right) \operatorname{erfc}\left(R_{i, m n p} E+j k / 2 E\right)}{R_{i, m n p}}+\right.} \\
&\left.\frac{\exp \left(-j k R_{i, m n p}\right) \operatorname{erfc}\left(R_{i, m n p} E-j k / 2 E\right)}{R_{i, m n p}}\right]
\end{aligned}
$$


Here the coefficient $A_{i}^{z z}$ is defined by

$A_{i}^{z z}=\left\{\begin{array}{ll}+1, & i=0,1,6,7 \\ -1, & i=2,3,4,5\end{array}\right.$,

The length $R_{i, m n p}$ represents the distance between a source and its mirror sources at $\mathbf{r}^{\prime}=\left(x^{\prime}, y^{\prime}, z^{\prime}\right)$ to an observation point at $\mathbf{r}=(x, y, z)$. It is given by

$$
\begin{aligned}
& R_{i, m n p}= \\
& \quad \sqrt{\left(X_{i}+2 m a\right)^{2}+\left(Y_{i}+2 n b\right)^{2}+\left(Z_{i}+2 p c\right)^{2}},
\end{aligned}
$$

with

$X_{i}=\left\{\begin{array}{ll}x-x^{\prime}, & i=0,1,2,3 \\ x+x^{\prime}, & i=4,5,6,7\end{array}\right.$,

$Y_{i}=\left\{\begin{array}{ll}y-y^{\prime}, & i=0,1,4,5 \\ y+y^{\prime}, & i=2,3,6,7\end{array}\right.$,

$Z_{i}=\left\{\begin{array}{ll}z-z^{\prime}, & i=0,2,4,6 \\ z+z^{\prime}, & i=1,3,5,7\end{array}\right.$.

The components $k_{0 x}, k_{0 y}$, and $k_{0 z}$ are defined by the vector $\mathbf{k}_{0}$ according to

$\mathbf{k}_{o}:=\left(k_{0 x}, k_{0 y}, k_{0 z}\right):=(m \pi / a, n \pi / b, p \pi / c)$.

Finally, we introduced in Eq. (6) the complementary error function $\operatorname{erfc}(z)$.

The term $G_{z z 1}^{A}$ in Eq. (5) constitutes a mode part while the term $G_{z z 2}^{A}$ in Eq. (6) constitutes a ray part. The a priori undetermined parameter $E$ adjusts the contributions of each of both terms to the sum in Eq. (4). Increasing $E$ makes $G_{z z 1}^{A}$ contribute more to the sum and decreases the influence of $G_{z z 2}^{A}$ while decreasing $E$ makes $G_{z z 2}^{A}$ contribute more to the sum and decreases the influence of $G_{z z 1}^{A}$. From a numerical point of view it is best to choose $E$ such that the decay of both series is balanced.

Let us plot the Green's function $G_{z z}^{A}\left(z, z^{\prime}\right)$ for illustration. We characterize the dimensions of the cavity by $a=b=$ $c=1$ and introduce a line within this cube which is given by $x=a / 2, y=b / 2$, and $z \in[c / 4,3 c / 4]$. As observation point we choose $\mathbf{r}=(a / 2, b / 2, c / 2)$ and take source points $\mathbf{r}^{\prime}=\left(a / 2, b / 2, z^{\prime}\right)$ with $z^{\prime} \in[c / 4,3 c / 4]$. We also need to specify a wave number. We choose for this example $k=$ $2 \pi(1-j /(2 Q)) /(2 / \sqrt{6})$. This corresponds to a resonance frequency of the cavity with $\lambda=2 / \sqrt{6}$. The letter $Q$ denotes the quality factor.

It is seen in the upper diagram of Fig. 2 that for a relatively low quality factor of $Q=40$ the Green's function of the cavity resembles the Green's function of free space. If we increase the quality factor it is seen that a rather extended part of an oscillation builds up. This is due to the choice of wavenumber which corresponds to a resonance frequency of the cavity. Larger quality factors, which correspond to fewer losses, lead to larger amplitudes that can arbitrarily exceed
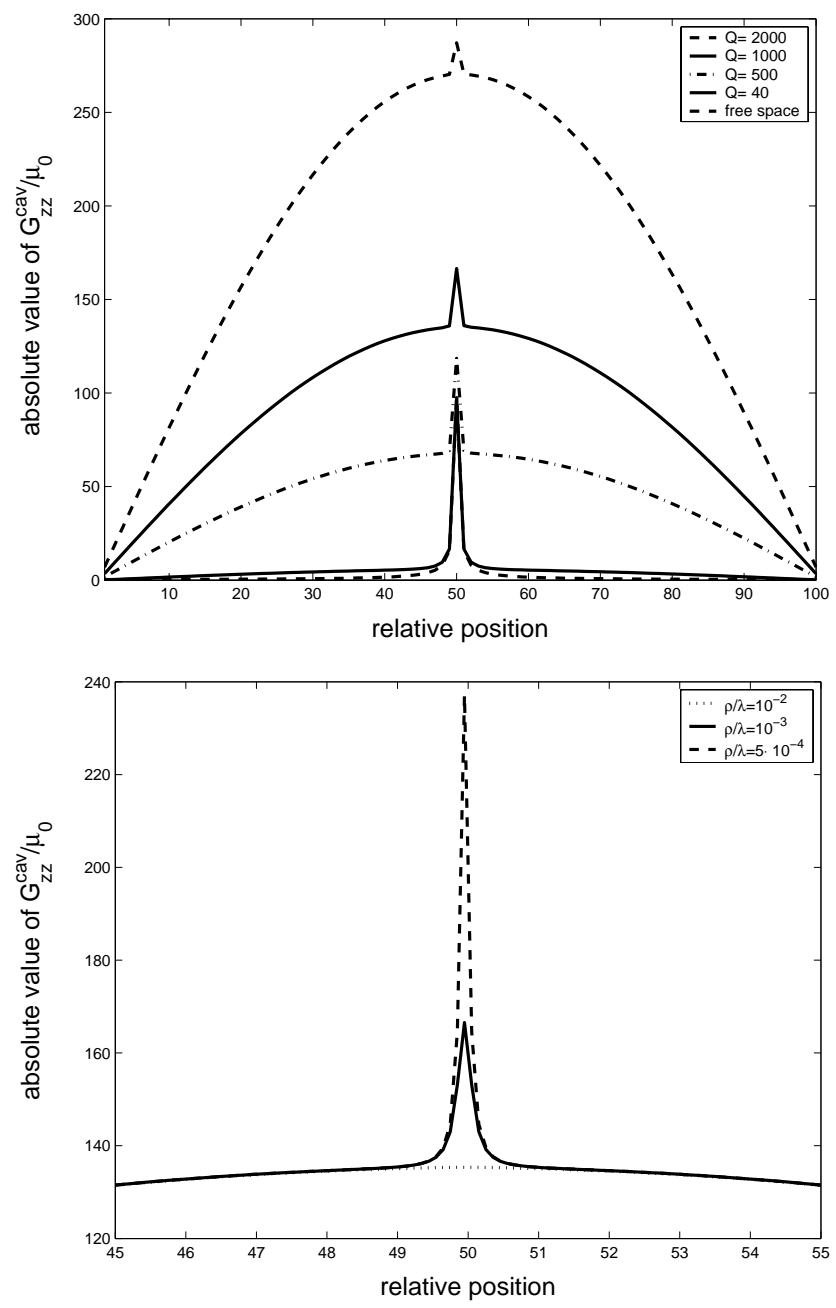

Fig. 2. Plot of the absolute value of the function $G_{z z}^{A}\left(\frac{c}{2}, z^{\prime}\right) / \mu_{0}$. The upper diagram displays plots for various quality factors $Q$ and a fixed radius which is given by $\rho / \lambda=10^{-3}$. The relative position relates to the interval $[\mathrm{c} / 4,3 \mathrm{c} / 4]$. The lower diagram focuses on the observation point at relative position 50. The corresponding three plots are obtained by keeping the quality factor fixed at a value of $Q=1000$ and using different values for the ratio $\rho / \lambda$.

the peak in the source region of free space which, in turn, is determined by the antenna radius. Smaller quality factors lead to an decrease of the oscillation such that in this case the curve resembles the situation of free space. The same decrease is observed if we shift the frequency off resonance. That the peak in the source region is indeed triggered by the wire radius is shown in the lower diagram of Fig. 2. There a fixed quality factor $Q=1000$ is chosen. It is seen that on top of the oscillation we have no peak for a relatively large antenna radius but can generate arbitrarily large peaks by choosing the antenna radius to be smaller and smaller.

From this short investigation of the cavities Green's function we may close this section by the following simple statement: Cavity effects become important close to resonance and for high quality factors. The actual value of a "high" quality factor is determined by the singular behavior in the 


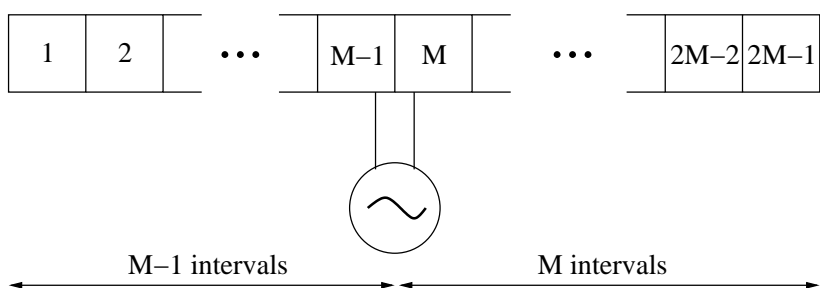

Fig. 3. Subdivision of the dipole antenna in $2 M-1$ equally spaced intervals of length $h=L /(2 M-1)$. The slice generator is located between the $M-1$ th and $M$ th interval.

source region: If the oscillations in the Green's function, that are due to a resonance, become comparable in amplitude to the peak in the source region, then it is expected that cavity effects will play a major role in the electromagnetic coupling process.

\section{Method of moments solution and numerical results}

We now solve Halléns Eq. (1) by the method of moments. To this end we subdivide the dipole antenna in $2 M-1$ equally spaced intervals of length $h=L /(2 M-1)$, with a delta gap generator between the $(M-1)$ th and $M$ th interval, compare Fig. 3. This introduces a slight asymmetry which is acceptable if $h$ is sufficiently small.

As basis functions we use pulse functions $P_{k}(z)$. For fixed $k$ the function $P_{k}(z)$ equals unity within the $k$ th interval and is zero otherwise. The unknown electric current is expanded according to

$I(z) \approx \sum_{k=1}^{2 M-1} \alpha_{k} P_{k}(z)$

Inserting this expansion in Eq. (1) and using the midpoints $z_{k}$ of the $2 M-1$ intervals as matching points leads to a linear system of $2 M-1$ equations for the unknowns $\alpha_{2} \ldots \alpha_{2 M-2}$, A. The coefficients $\alpha_{1}, \alpha_{2 M-1}$ are set to zero in order to fulfill the boundary condition of vanishing current at the ends of the antenna. The system of equations explicitly is given by

$\sum_{k=1}^{2 M-1} A_{j k} a_{k}=b_{j}$

with

$a_{k}:=\left(\alpha_{2}, \ldots, \alpha_{2 M-2}, A\right)_{k}, \quad b_{j}:=-\frac{j V_{0}}{2 \eta} \sin \left(k\left|z_{j}\right|\right)$,

and

$A_{j(2 M-1)}:=\frac{j}{\eta} \cos \left(k z_{j}\right), \quad A_{j k}:=\int_{z_{k}-h / 2}^{z_{k}+h / 2} G_{z z}^{A}\left(z_{j}, z^{\prime}\right) d z^{\prime}$.

for $k=1 \ldots 2 M-2$.

The matrix elements $A_{j k}$ of Eq. (16) can either be numerically evaluated or analytically approximated. Once this is done the linear Eq. (14) is straightforwardly solved such that

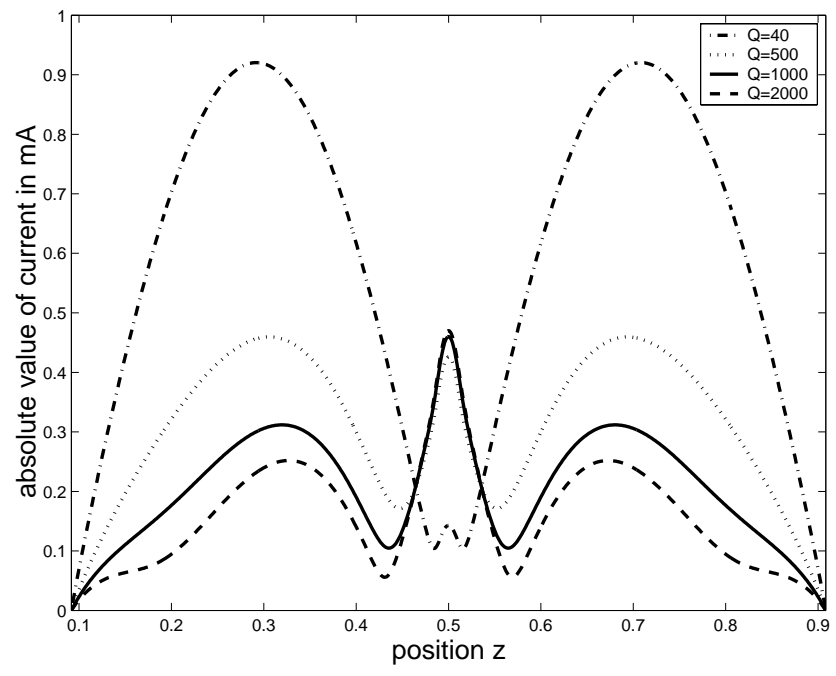

Fig. 4. Distribution of the absolute value of the current on a dipole antenna within a rectangular cavity. The normalized length is $L / \lambda=1$ and the antenna radius is given by $\rho / \lambda=10^{-3}$.

the current $I(z)$ is obtained via Eq. (13). In this way we now calculate the current on a dipole antenna for various values of the quality factor. The antenna is placed at $x=a / 2$, $y=b / 2$, and extends along $z \in[-L / 2+c / 2, L / 2+c / 2]$. The choice of parameters is the same as the one that was used to obtain the upper diagram of Fig. 2. Furthermore, we normalize the slice generator and set $V_{0}=1$. The corresponding results are displayed in Fig. 4.

For the low quality factor $Q=40$ we expect in view of the upper diagram of Fig. 2, which shows a dominating source singularity, that the current distribution of the antenna within the cavity is similar to that in free space, even though we excite a resonance frequency of the cavity. This is indeed the case: The current distribution is approximately sinusoidal, with a deviation at the center of the antenna which can be attributed to the slice generator. This feature is well-known from the method of moment analysis of a dipole antenna in free space (Elliott, 1981). If we increase the quality factor the resulting current distribution no longer resembles the current distribution of free space but is characterized by an increasing maximum at the center of the dipole antenna, while the former maxima of the sinusoidal distribution decrease. It is clear that this current distribution corresponds to a dominating resonance.

With this computation of the current distribution the boundary value problem of an active dipole antenna within a rectangular cavity is completely solved. The electromagnetic field inside the cavity can straightforwardly be obtained by means of the cavity's Green's function.

In summary, there are two major points that can be seen from this example: First, it is decisive to find an efficient representation of the electromagnetic Green's function if coupling phenomena within a cavity are considered. The efficiency and accuracy of the proposed representation in Sect. 2, which is valid for a rectangular cavity, is of orders of magni- 
tudes better than the standard mode representation. It is this circumstance that makes a method of moment solution feasible. Second, it is important to understand the dominating influence of both the source singularity and the resonances. Both types of singularities are triggered by a certain parameter. While a dominating source singularity leads to an electromagnetic coupling which resembles that of free space it is a dominating resonance which exhibits cavity effects. This insight is also useful for analytical calculations that are based on iteration or perturbative methods, where dominating firstorder solutions are required.

Acknowledgements. The author would like to thank Dr. H.-G. Krauthäuser, Prof. J. Nitsch, and Dr. S. Tkachenko for useful discussions.

\section{References}

Elliott, R. S.: Antenna theoryand design, Prentice-Hall, Englewood Cliffs 1981.

Ewald, P. P.: Die Berechnung optischer und elektrostatischer Gitterpotentiale, Annalen der Physik, 64, 253-268, 1921.

Felsen, L. P., (Ed): Hybrid Formulation of Wave Propagation and Scattering, Martinus Nijhoff, Dordrecht, 1984.
Gronwald, F., Nitsch, J., and Tkachenko, S: Hybrid representation methods for the efficient analysis of antenna coupling within cavities, EMC 2002 - Proc. of the Sixteenth International Wroclaw Symposium on Electromagnetic Compatibility, Wroclaw, Poland, 109-114, June 2002.

Gronwald, F.: Efficient Calculation of the Green's Function for Lossy Rectangular Cavities, submitted to IEEE Microwave Guided Wave Lett., 2002.

Lee, K. S. H., (Ed): EMP Interaction: Principles, Techniques, and Reference Data, revised printing, Taylor \& Francis, Washington D.C., 1995.

Nakano, H.: Antenna Analysis using Integral Equations, Analysis Methods for Electromagnetic Wave Problems, Volume Two, E. Yamashita (ed.) Artech House, Boston, 1996.

Park, M.-J., Park, J., and Nam, S.: Efficient Calculation of the Green's Function for the Rectangular Cavity, IEEE Microwave and Guided Wave Letters, 124-126, 8 March 1998.

Tai, C.-T.: Dyadic Green Functions in Electromagnetic Theory, IEEE Press, New York, 1994.

Tesche, F. M., Ianoz, M. V., and Karlsson, T.: EMC Analysis Methods and Computational Methods, John Wiley \& Sons, New York, 1997.

Wu, D. I. and Chang, D. C.: A Hybrid Representation of the Green's Function in an Overmoded Rectangular Cavity, IEEE Trans. on MTT, 36, 1334-1342, September 1988. 